\title{
Serial Assays of QuantiFERON-TB Gold In-Tube and QuantiFERON-TB Gold-Plus in Subjects Exposed to Patients with Active Tuberculosis
}

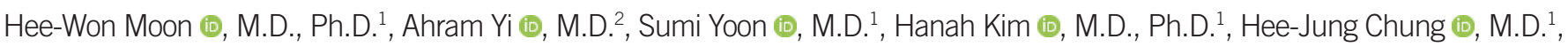

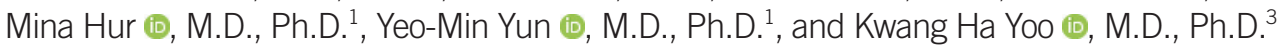

${ }^{1}$ Department of Laboratory Medicine, Konkuk University School of Medicine, Seoul, Korea; ${ }^{2}$ Department of Laboratory Medicine, Green Cross Laboratories, Yongin, Korea; ${ }^{3}$ Department of Internal Medicine, Konkuk University School of Medicine, Seoul, Korea

\section{Dear Editor,}

The detection and management of subjects with latent tuberculosis infection (LTBI) are important steps to control and decrease the incidence of tuberculosis (TB). Exposure to patients with active TB is one of the most important transmission routes of LTBI [1]. Interferon-gamma (IFN- $\gamma$ ) release assays (IGRAs), in particular, the QuantiFERON-TB Gold In-Tube assay (QFT; QIAGEN, Germantown, MD, USA), have been widely used for investigating subjects exposed to patients with active TB. However, high reversion rates and low reproducibility of QFT have also been documented [2].

The new version of QFT, QuantiFERON-TB Gold-Plus (QFTPlus, QIAGEN), might provide higher sensitivity for detection of early infection in subjects exposed to patients with active TB, owing to its ability to assess both CD8+ and CD4+ T cell-mediated immune responses $[3,4]$. Since there is sparse data on QFT and QFT-Plus in subjects exposed to patients with active TB $[5,6]$, we investigated the results of serial assays of QFT and QFT-Plus in such subjects. From December 2016 to November 2018, 69 subjects exposed to patients with active TB (including 57 households and 12 occupational exposures; median age [in- terquartile range, IQR], 35 years [27-50 years]) were enrolled at Konkuk University Medical Center (KUMC), Seoul, Korea. We excluded subjects who had a history of a positive QFT or tuberculin skin test (TST) result. This study was approved by the Institutional Review Board of KUMC (KUH1200076), and informed consent was obtained from all enrolled subjects.

Both QFT and QFT-Plus were performed within eight weeks of first exposure as the first assessment, with a median (IQR) of 14 (6-30) days after exposure. Follow-up assays were performed in 39 subjects at eight weeks after the first assessment (i.e., second assessment). If there were discrepancies between the first and second assessments, a third assessment was performed after eight weeks, when possible.

Peripheral blood (1 mL; Nil, QFT TB antigen, QFT-Plus TB1, TB2 antigen [CD4+ $T$ cell response and both CD4+ and CD8+ $\mathrm{T}$ cell response, respectively]) was collected in each specialized tube. The samples were immediately incubated at $37^{\circ} \mathrm{C}$ for 16 24 hours, and the separated plasma was stored at $4^{\circ} \mathrm{C}$. QFT and QFT-Plus were performed according to the manufacturer's instructions. For QFT, the results are considered positive when the TB antigen minus Nil IFN- $\gamma$ concentration is $\geq 0.35 \mathrm{IU} / \mathrm{mL}$
Received: November 29, 2019

Revision received: January 13, 2020

Accepted: March 3, 2020

Corresponding author: Hee-Won Moon, M.D., Ph.D.

Department of Laboratory Medicine, Konkuk University School of Medicine Konkuk University Medical Center, 120-1 Neungdong-ro, Gwangjin-gu, Seoul 05030, Korea

Tel: +82-2-2030-5583, Fax: +82-2-2030-5587, E-mail: hannasis@kuh.ac.kr

\section{(c) (1) $(9$}

(C) Korean Society for Laboratory Medicine

This is an Open Access article distributed under the terms of the Creative Commons Attribution Non-Commercial License (https://creativecommons.org/licenses/by-nc/4.0) which permits unrestricted non-commercial use, distribution, and reproduction in any medium, provided the original work is properly cited. 
and $\geq 25 \%$ of the Nil value. The QFT-Plus is interpreted as positive when the TB antigen tube (TB1 or TB2) minus Nil IFN- $\gamma$ concentration is $\geq 0.35 \mathrm{IU} / \mathrm{mL}$ and $\geq 25 \%$ of the Nil value. Concordance between the results of the two assays was measured using Cohen's Kappa, and statistical analysis was performed using MedCalc Statistical Software (version 17.2; MedCalc Software, Ostend, Belgium).

The agreement between QFT and QFT-Plus results was strong (110 coupled tests, kappa $=0.857,95 \%$ confidence interval $=0.746-0.967$, overall concordance rate $=94.5 \%$ ). The positive rate of QFT and QFT-Plus at the first assessment was $24.6 \%$ (17/69) and 26.1\% (18/69), respectively. Prophylactic treatment was performed in eight subjects between the first and second assessments. The positive rate of QFT and QFT-Plus at the second assessment was 23.1\% (9/39) and 28.2\% (11/39), respectively. The IFN- $\gamma$ concentrations of the TB antigen minus Nil tube in QFT and QFT-Plus are shown in Table 1. The QFT-
Plus TB2 values were significantly higher in the second assessment than in the first assessment $(P=0.032$, Wilcoxon signed rank test). However, the QFT TB and QFT-Plus TB1 values were not significantly different between the first and second assessments.

Among the 39 subjects with available serial assay results, the conversion rate (negative to positive) of QFT and QFT-Plus was $3.5 \%(1 / 29)$ and $10.7 \%(3 / 28)$, respectively, and the reversion rate (positive to negative) was $20.0 \%(2 / 10)$ and $27.3 \%(3 / 11)$, respectively. The inconsistent results between QFT and QFTPlus or between the first and follow-up assessments are shown in Table 2. Samples from two subjects (C22 and C61) showed positivity only in QFT-Plus TB2, suggesting that TB2 might be converted earlier in cases of recent exposure. In particular, samples from C22 showed only TB2 positivity consistently on the first, second, and third assessments, and the TB-Nil, TB1$\mathrm{Nil}$, and TB2-Nil values showed an increasing trend (except TB

Table 1. Results of QuantiFERON-TB Gold In-Tube (TB), QuantiFERON-TB Gold-Plus TB1 (TB1), and QuantiFERON-TB Gold-Plus TB2 (TB2) minus Nil values in the first and second assessments

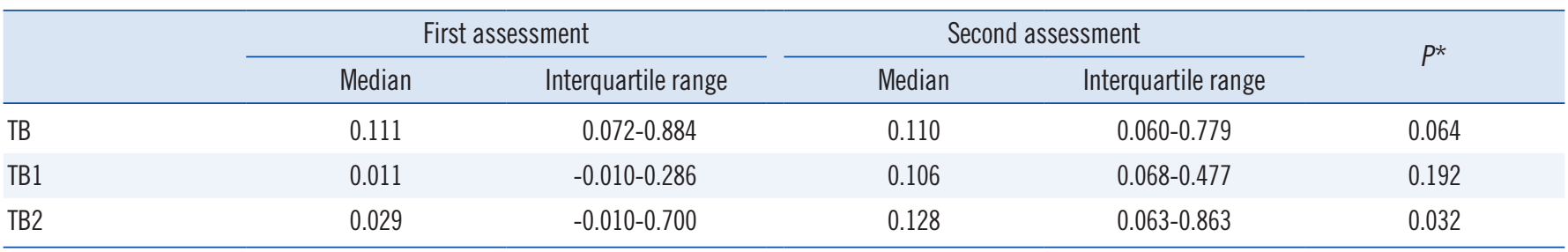

${ }^{*} P$ was determined by the Wilcoxon signed rank test.

Abbreviations: TB, TB minus Nil; TB1, TB1 minus Nil; TB2, TB2 minus Nil.

Table 2. Inconsistent results of QuantiFERON-TB Gold In-Tube (TB), QuantiFERON-TB Gold-Plus TB1 (TB1), and QuantiFERON-TB GoldPlus TB2 (TB2) or between the first and follow-up assessments

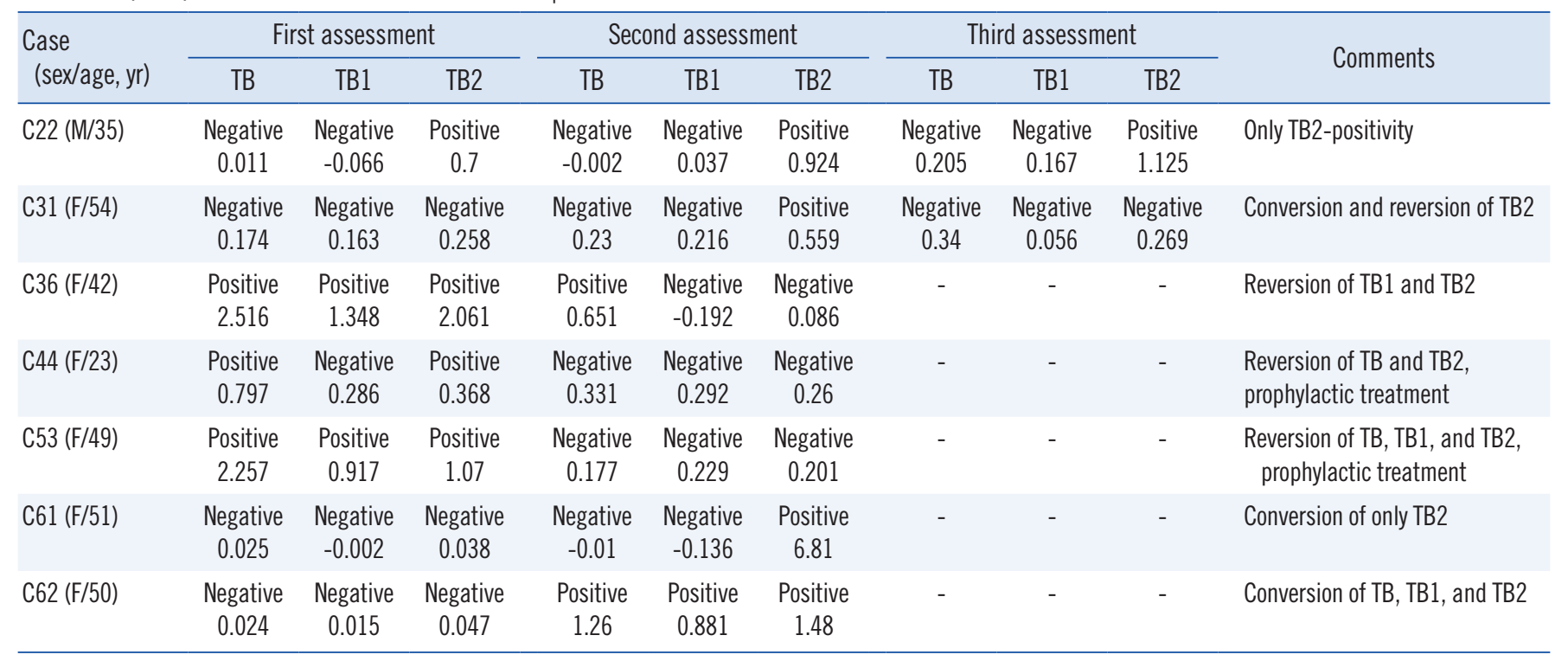

Abbreviations: M, Male; F, Female; TB, TB minus Nil; TB1, TB1 minus Nil; TB2, TB2 minus Nil. 
at the second assessment), indicating an ongoing conversion process. Samples from C61 showed an abrupt increase in TB2Nil; thus, a follow-up assay would be helpful for accurate interpretation, although it could not be performed in this study. Another subject (C62) showed a positive result only at the second assessment; the first assessment was too early (at four days after exposure), and a second assessment was, therefore, necessary in this subject. Recent findings suggest more delayed conversion with IGRA than with the TST, whereas QFT-Plus showed earlier conversion than QFT [1, 7, 8]. Reversion was observed in four subjects (C31, C36, C44, and C53), including two with QFT-TB (C44 and C53), two with QFT-Plus TB1 (C36 and C53), and four with TB2 (C31, C36, C44, and C53). Prophylactic treatment might explain the reversion in two subjects (C44 and C53) [9]. Reversion in the other two subjects could be related to natural clearing, an unstable immune response, or assay-related variability [10] due to pre-analytical, analytical, post-analytical, manufacturing, and immunological factors [2]. Since we could not perform a baseline QFT, the possibility that infection in some subjects occurred before exposure to patients with active TB cannot be ruled out. We tried to decrease this possibility by excluding subjects with a history of a positive QFT or TST result.

Despite the limited sample size, we showed serial results of QFT and QFT-Plus in subjects exposed to patients with active TB. Since QFT-Plus TB2 seems to be converted earlier, followup assays would be helpful before starting prophylactic treatment, owing to the possibility of reversion and delayed conversion. Moreover, assessment should be done within enough time to allow for maturation of the immune response, and detailed guidelines should be prepared using additional data.

\section{ACKNOWLEDGEMENTS}

QIAGEN provided the QFT-Plus kits for this study.

\section{AUTHOR CONTRIBUTIONS}

MHW designed the study, analyzed the data, and wrote the manuscript. YA, YS, and YKH participated in subject enrollment, research protocol and analysis. $\mathrm{KH}, \mathrm{CHJ}, \mathrm{HM}$, and YYM reviewed the manuscript. All authors read and approved the final manuscript.

\section{CONFLICTS OF INTEREST}

\section{RESEARCH FUNDING}

None declared.

\section{ORCID}

Hee-Won Moon

Ahram Yi

Sumi Yoon

Hanah Kim

Hee-Jung Chung

Mina Hur

Yeo-Min Yun

Kwang Ha Yoo

https://orcid.org/0000-0001-9509-6073

https://orcid.org/0000-0002-3107-936X

https://orcid.org/0000-0001-7529-1613

https://orcid.org/0000-0002-3266-638X

https://orcid.org/0000-0002-1479-0731

https://orcid.org/0000-0002-4429-9978

https://orcid.org/0000-0002-5485-8331

https://orcid.org/0000-0001-9969-2657

\section{REFERENCES}

1. Barcellini L, Borroni E, Brown J, Brunetti E, Campisi D, Castellotti PF, et al. First evaluation of QuantiFERON-TB Gold Plus performance in contact screening. Eur Respir J 2016;48:1411-9.

2. Banaei N, Gaur RL, Pai M. Interferon-gamma release assays for latent tuberculosis: what are the sources of variability? J Clin Microbiol 2016; 54:845-50

3. Rozot V, Patrizia A, Vigano S, Mazza-Stalder J, Idrizi E, Day CL, et al. Combined use of Mycobacterium tuberculosis-specific CD4 and CD8 Tcell responses is a powerful diagnostic tool of active tuberculosis. Clin Infect Dis 2015;60:432-7.

4. Moon HW, Gaur RL, Tien SS, Spangler M, Pai M, Banaei N. Evaluation of QuantiFERON-TB Gold-Plus in health care workers in a low-incidence setting. J Clin Microbiol 2017;55:1650-7.

5. Gonzślez-Moreno J, García-Gasalla M, Gallego-Lezaun C, Fernández-Baca V, Mir Viladrich I, Cifuentes-Luna C, et al. Role of QuantiFERON(®)TB Gold In-Tube in tuberculosis contact investigation: experience in a tuberculosis unit. Infect Dis (Lond) 2015;47:244-51.

6. Ferrarini MA, Spina FG, Weckx LY, Lederman HM, De Moraes-Pinto MI. Rate of tuberculosis infection in children and adolescents with household contact with adults with active pulmonary tuberculosis as assessed by tuberculin skin test and interferon-gamma release assays. Epidemiol Infect 2016;144:712-23.

7. Lee SW, Oh DK, Lee SH, Kang HY, Lee CT, Yim JJ. Time interval to conversion of interferon-gamma release assay after exposure to tuberculosis. Eur Respir J 2011;37:1447-52.

8. Ribeiro-Rodrigues R, Kim S, Coelho da Silva FD, Uzelac A, Collins L, Palaci $\mathrm{M}$, et al. Discordance of tuberculin skin test and interferon gamma release assay in recently exposed household contacts of pulmonary TB cases in Brazil. PLoS One 2014;9:e96564.

9. Zhang H, Xin H, Li X, Li H, Li M, Feng B, et al. Reversion of QuantiFERON-TB Gold In-Tube test in individuals with and without prophylactic treatment for latent tuberculosis infection: a systematic review and meta-analysis. J Infect 2018;77:276-82.

10. Pai M, Denkinger CM, Kik SV, Rangaka MX, Zwerling A, Oxlade O, et al. Gamma interferon release assays for detection of Mycobacterium tuberculosis infection. Clin Microbiol Rev 2014;27:3-20.

None declared. 